\title{
Rydberg spectra of ArH. Bound-bound interactions, predissociation, and radiative lifetimes of the Rydberg states
}

\author{
G. Theodorakopoulos and I. D. Petsalakis. \\ Theoretical and Physical Chemistry Institute, National Hellenic Research Foundation, \\ 48, Vas. Constantinou Ave., 11635 Athens, Greece
}

(Received 24 January 1994; accepted 14 March 1994)

\begin{abstract}
Bound-bound ${ }^{2} \Sigma^{+}-{ }^{2} \Sigma^{+}$and ${ }^{2} \Sigma^{+}-{ }^{2} \Pi$ interactions and their effect on the rotational-vibrational levels of Rydberg states of ArH/ArD have been investigated. Strong interactions have been obtained for the $B^{2} \Pi-C^{2} \Sigma^{+}, D^{2} \Sigma^{+}-E^{2} \Pi$, and $E^{2} \Pi-1^{2} \Delta$ pairs of states in accord with recent. experimental analyses of Rydberg spectra of ArD. Predissociation lifetimes of $A^{2} \Sigma^{+}, C^{2} \Sigma^{+}$, and $D^{2} \Sigma^{+}$as well as transition energies and radiative lifetimes for Rydberg states up to $4 f$ have been also calculated.
\end{abstract}

\section{INTRODUCTION}

The analysis of the observed Rydberg spectra of ArH seems to be a rather difficult task. In terms of the potential energy curves, at least at short internuclear distances, the electronic states of ArH give the typical picture of a Rydberg molecule; ${ }^{1}$ a repulsive ground state (except for a van der Waals minimum at large $R$ ) and bound Rydberg states with deep minima and equilibrium internuclear distance near that of the ground state of $\mathrm{ArH}^{+}$, the core cation..$^{2-4}$ A number of ${ }^{2} \Pi \rightarrow A^{2} \Sigma^{+}$transitions have been reported, ${ }^{5}$ following the first spectrum of ArH which was obtained in 1970 (Ref. 6) while recently three new bands of ArD have been analyzed in terms of transitions involving a $p\left({ }^{2} \Sigma,{ }^{2} \Pi\right)$ state, presumably a combination of the $C^{2} \Sigma^{+}$and $B^{2} \Pi$ states ${ }^{4}$ of ArH. Other experimental work, which has been devoted to the determination of the lifetimes of Rydherg states of ArH and $\operatorname{ArD}$, has resulted in some very interesting findings regarding the lifetime of the $E^{2} \Pi$ state of $\mathrm{ArD}$, obtained from the $E^{2} \Pi \rightarrow A^{2} \Sigma^{+}$emission; ${ }^{8}$ a longer lifetime is obtained from the $Q$ branch than those obtained from the $P$ and $R$ branches of the above emission. The authors ${ }^{8}$ have attributed the above observations to a depletion of the $E^{2} \Pi^{+}$population (which would give rise to the $P$ and $R$ branches in a ${ }^{2} \Pi \rightarrow{ }^{2} \Sigma^{+}$transition) via a rotational-electronic coupling with the presumably predissociating state $D^{2} \Sigma^{+}$. Furthermore, they have observed a decrease in intensity for $N>16$ in all branches $(P, Q, R)$, indicating a further nonradiative channel for the decay of $E^{2} \Pi$ in ArH and ArD. While it has not been suggested by the above authors, a different radiative channel may be provided by the $1^{2} \Delta$ state, via a rotational electronic coupling $E^{2} \Pi-1^{2} \Delta$. The $1^{2} \Delta$ state is not predissociating but it decays radiatively to $B{ }^{2} \Pi$ and it could serve to remove population from the $E^{2} \Pi \rightarrow A^{2} \Sigma^{+}$transition.

Theoretical treatments of the Rydberg states of ArH are generally in good agreement with experiment, as far as the energy levels are concerned. ${ }^{3,4}$ The predissociation of the ${ }^{2} \Pi$ states and of $A^{2} \Sigma^{+}$by the repulsive ground state, $X^{2} \Sigma^{+}$(via rotational-electronic coupling and radial coupling, respectively), has been also studied and it has been established that predissociation of the ${ }^{2} \Pi$ states of $\operatorname{ArH} / \mathrm{ArD}$ by $X^{2} \Sigma^{+}$is negligible $4^{4}$ while predissociation of $A^{2} \Sigma^{+}$is the exclusive mode of decay, as indicated by the relative magnitudes of the rates of predissociation and radiative dissociation. ${ }^{3,9}$ In addition to the above, theoretical values for the radiative lifetimes of a number of Rydberg states of ArH have been reported ${ }^{9}$ where, for the higher states, emissions to $X^{2} \Sigma^{+}, A^{2} \Sigma^{+}$, and $B^{2} \Pi$ only have been considered. Comparison of the theoretical values with the available experimental data showed good agreement for the lifetime of the $B^{2} \Pi$ state, and for the $E^{2} \Pi$ state with the value reported for $N \leqslant 16$, in $\mathrm{ArH}^{8}$

In view of the recent experimental analyses, it has been necessary to extend our theoretical work along the following lines: (a) calculation of rotational-electronic coupling between the bound ${ }^{2} \Sigma^{+}$and ${ }^{2} \Pi$ states and in an effort to establish whether the $C-B$ and $D-E$ interactions are sufficiently strong to support the arguments upon which the experimental analyses ${ }^{7,8}$ have been performed. In addition, the rotational-electronic coupling $E^{2} \Pi-1^{2} \Delta$ must be examined as a possible mechanism for the depletion of population from the higher rotational levels of $E^{2} \Pi$. This interaction is also of interest in view of the suggestion ${ }^{10}$ of the formation of a $d(\Sigma, \Pi, \Delta)$ complex by the $D^{2} \Sigma^{+}, E^{2} \Pi$, and $1^{2} \Delta$ states. (b) Examination by actual calculations of the assumed predissociation of the $C^{2} \Sigma^{+}$and $D^{2} \Sigma^{+}$states of ArH by $X^{2} \Sigma^{+}$as the predominant mode of decay of these states, since the recently reported experimental work ${ }^{7}$ indicates the existence of radiative transitions from the higher ${ }^{2} \Sigma^{+}$states, and (c) further examination of the radiative lifetimes of Rydberg states of ArH. In particular, it is required to include all possible radiative channels for decay in order to converge the theoretical values for the radiative lifetimes while generation of information regarding transitions from even higher-lying Rydberg states than those considered thus $\operatorname{far}^{4,9}$ is also desirable.

\section{COMPUTATIONAL DETAILS}

In the present calculations four ${ }^{2} \Sigma_{i}^{+}\left({ }^{2} A_{1}\right)$, one ${ }^{2} \Delta\left({ }^{2} A_{1}\right)$, and three ${ }^{2} \Pi\left({ }^{2} B_{1}\right)$ states have been considered for the investigation of the nonradiative interactions. The $A O$ basis set was the same as in the previous work, ${ }^{9}$ where Rydberg functions have been included on hydrogen up to $4 p$ and on argon for $4 s, 4 p$, and $3 d$. The MRD-CI programs $s^{11,12}$ 
have been employed for the calculations. For the ${ }^{2} A_{1}$ states (four ${ }^{2} \Sigma^{+}$and one ${ }^{2} \Delta$ ) the $C I$ calculations employed 43 reference configurations and selection for 5 roots with a threshold of $5 \mu \mathrm{H}$. For the ${ }^{2} B_{1}$ states $\left(B^{2} \Pi, E^{2} \Pi\right.$, and $\left.3^{2} \Pi\right), 34$ reference configurations and selection for 3 roots with $3.0 \mu \mathrm{H}$ threshold have been employed. The CI spaces varied between 15000 and 18000 configuration functions, depending on the value of the internuclear distance $R$. The calculations have been carried out for 17 values of $R$, in the interval from 1.6 to $5.0 \mathrm{bohr}$. At each point, in addition to the energy, dipole transition moments for all the possible transitions between the above states as well as rotational electronic couplings between the ${ }^{2} \Sigma^{+}$and the ${ }^{2} \Pi$ states have been determined. Furthermore, for the ${ }^{2} \Sigma^{+}$states, radial coupling matrix elements have been computed at each point $R$ by carrying out a second Cl calculation at $R+0.001$ bohr, with the center placed at the center of mass of ArH. Radial coupling matrix elements have been obtained for the pairs of states, $X-A, X-C, X-D$ and also $A-C, A-D$, and $C-D$. Although the $X^{2} \Sigma^{+}-A^{2} \Sigma^{+}$interaction has been the subject of previous extensive calculations, ${ }^{9}$ which are beyond our computing capabilities to repeat for five ${ }^{2} A_{1}$ states, it is instructive to calculate it again under the present selection procedure, in order to check the precision of the theoretical results, regarding this type of predissociation. For a system of the size of ArH it is rather difficult to calculate the radial coupling matrix elements with good precision because the finite differences method..which is employed ${ }^{13}$ has been found to be rather sensitive to the particulars of the CI selection procedure.

The data generated as described above, have been employed in subsequent calculations of the predissociation rates and bound-bound interactions. The method and programs employed at this point have been described elsewhere. ${ }^{14-16}$

For the radiative lifetimes in addition to the above, further calculations have been carried out at a single geometry, with $R$ of 2.5 bohr, involving $12^{2} \Sigma^{+}, 3^{2} \Delta, 8^{2} \Pi$, and 1 ${ }^{2} \Phi$ Rydberg states. For these calculations, additional Rydberg functions have been included in the hydrogen basis, a set of $4 d$ functions with exponent 0.005 and a set of $4 f$ functions with exponent 0.008 . The $\mathrm{CI}$ procedures were as follows: for the ${ }^{2} \Sigma^{+}$and ${ }^{2} \Delta$ states, a reference set of 63 configurations and selection with respect to 9 roots (for the first $9^{2} A_{1}$ states) and eight roots (for states $9{ }^{2} A_{1}$ to $15^{2} A_{1}$ ) with a threshold of $5 \mu \mathrm{H}$. For the ${ }^{2} B_{1}$ states the reference space involved 48 configurations and selection was carried out for nine roots with $5 \mu \mathrm{H}$ threshold. From this single "vertical" calculation, radiative transition propabilities have been determined and radiative lifetimes of the Rydberg states, where transitions to all possible lower states up to $3{ }^{2} \Pi$ have been included. For Rydberg transitions this type of calculation is sufficient and vibrational averaging does not affect the results significantly.

\section{RESULTS AND DISCUSSION}

\section{A. Radiative lifetimes}

The potential energy curves of the states calculated in the present work (cf. Fig. 1) are not different from the pre-

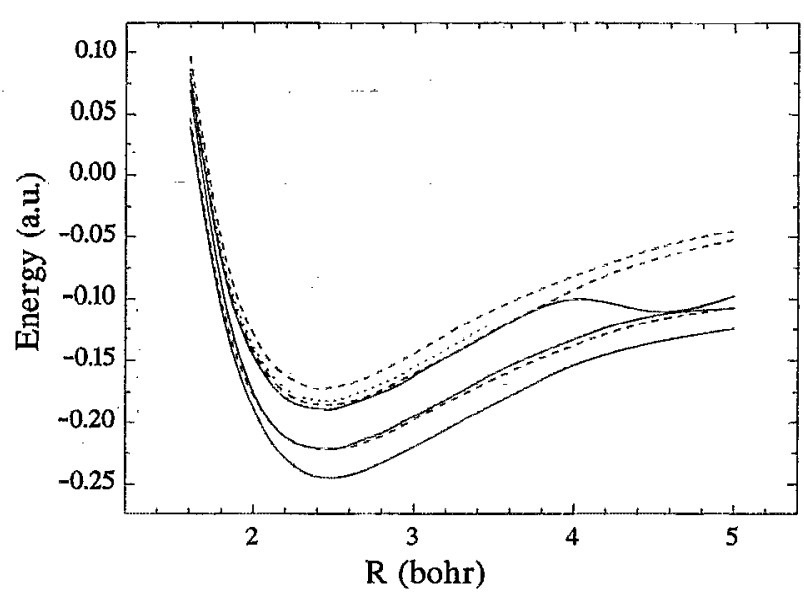

FIG. 1. Potential energy curves of the Rydberg states of ArH under consideration in the present work. Solid lines, ${ }^{2} \Sigma^{+}$states; dashed, ${ }^{2} \Pi$; dotted, ${ }^{2} \Delta$.

viously reported curves ${ }^{4}$ and, in general, quantitics related to the energy of the lower states (up to $5^{2} \Pi$ ) have differences within $0.05 \mathrm{eV}$ between previous treatments and the present. This is also demonstrated in Table I, where the energies of the excited states at $2.5 \mathrm{bohr}$ with respect to the corresponding energy of the ground state are listed. Thus the inclusion of the $4 d$ and $4 f$ Rydberg functions does not affect the lower-lying states significantly, while it has a significant effect on the position of the $6^{2} \Pi$ and $7^{2} \Pi$ (old numbering ${ }^{6}$. $5^{2} \Pi$ and $\left.6^{2} \Pi\right)$ states and introduces some new ${ }^{2} \Sigma^{+},{ }^{2} \Delta$,

TABLE I. Energies ${ }^{\natural}$ and radiative lifetimes of the electronic states of ArH at an internuclear distance of 2.5 bohr.

\begin{tabular}{lllc}
\hline \hline State & Atomic limit & \multicolumn{1}{c}{$\Delta E^{\mathrm{a}}(\mathrm{eV})$} & $\tau_{\text {rad }}(\mathrm{ns})$ \\
\hline$A^{2} \Sigma^{+}$ & $(2 s)$ & $3.34(3.23,3.32)$ & $1.73 \mu \mathrm{s}(2.3 \mu \mathrm{s})$ \\
$B^{2} \Pi$ & $(2 p)$ & $3.92(3.92)$ & $16.9(15.5)$ \\
$C^{2} \Sigma^{+}$ & $(2 p)$ & $4.01(3.97,3.98)$ & $7.42(7.2)$ \\
$D^{2} \Sigma^{+}$ & $\left(\mathrm{Ar}{ }^{1,3} P\right)$ & $4.90(4.86)$ & $35.0(498)$ \\
$E^{2} \Pi$ & $\left(\mathrm{Ar}{ }^{1,3} P\right)$ & $4.87(4.90)$ & $35.2(37.5)$ \\
$1^{2} \Delta$ & $(3 d)$ & $5.08(5.04)$ & $29(62)$ \\
$5^{2} \Sigma^{+}$ & $\left(\mathrm{Ar}{ }^{1,3} P\right)$ & $5.17(5.11)$ & $31(80)$ \\
$3^{2} \Pi$ & $\left(\mathrm{Ar}{ }^{1,3} P\right)$ & $5.27(5.26)$ & $42(46.8)$ \\
$6^{2} \Sigma^{+}$ & $(3 s)$ & $5.35(5.30)$ & 19 \\
$4^{2} \Pi$ & $(3 p)$ & 5.63 & $46(351)$ \\
$7^{2} \Sigma^{+}$ & $(3 p)$ & $5.66(5.62)$ & 225 \\
$8^{2} \Sigma^{+}$ & $(3 d)$ & 5.68 & 63 \\
$5^{2} \Pi$ & $(3 d)$ & $5.70(5.76)$ & 38 \\
$2^{2} \Delta$ & $(4 d)$ & 5.71 & 38 \\
$3^{2} \Delta$ & $(4 f)$ & 5.72 & 52 \\
$1^{2} \Phi$ & $(4 f)$ & 5.73 & 57 \\
$9^{2} \Sigma^{+}$ & $(4 s)$ & 5.70 & 42 \\
$10^{2} \Sigma^{+}$ & $(4 p)$ & 5.78 & 105 \\
$6^{2} \Pi$ & $(4 p)$ & $5.79(5.90)$ & 115 \\
$11^{2} \Sigma^{+}$ & $(4 d)$ & 6.02 & 109 \\
$7^{2} \Pi$ & $(4 d)$ & $6.23(6.37)$ & 123 \\
$12^{2} \Sigma^{+}$ & $(4 f)$ & 6.38 & 18 \\
$8^{2} \Pi$ & $(4 f)$ & 6.49 & 35 \\
\hline \hline
\end{tabular}

With respect to the energy of $X^{2} \Sigma^{+}$at $2.5 \mathrm{bohr}$, values in brackets are previous theoretical results, Ref. 6 for $\Delta E$, and Ref. 9 for $\tau_{\text {rad }}$.

${ }^{b}$ Hydrogen atom state unless otherwise indicated. 
TABLE II. Vertical radiative transition probabilities $\left(\mathrm{s}^{-1}\right)^{\mathrm{a}}$ for the Rydberg states of $\mathrm{ArH}$ at an internuclear distance of 2.5 bolır.

\begin{tabular}{lcccccccccc}
\hline \hline Lower state & & & & & & & & \\
Upper state & $X^{2} \Sigma^{+}$ & $A^{2} \Sigma^{+}$ & $B^{2} \Pi$ & $C^{2} \Sigma^{+}$ & $D^{2} \Sigma^{+}$ & $E^{2} \Pi$ & $1^{2} \Delta$ & $5^{2} \Sigma^{+}$ & $6^{2} \Sigma^{+}$ & $3^{2} \Pi$ \\
\hline$A^{2} \Sigma^{+}$ & $0.57(6)$ & $\ldots$ & $\ldots$ & $\ldots$ & $\ldots$ & $\ldots$ & $\ldots$ & $\ldots$ & $\ldots$ & $\ldots$ \\
$B^{2} \Pi$ & $0.56(8)$ & $0.33(7)$ & $\ldots$ & $\ldots$ & $\ldots$ & $\ldots$ & $\ldots$ & $\ldots$ & $\ldots$ & $\ldots$ \\
$C^{2} \Sigma^{+}$ & $0.13(9)$ & $0.22(7)$ & $\ldots$ & $\ldots$ & $\ldots$ & $\ldots$ & $\ldots$ & $\ldots$ & $\ldots$ & $\ldots$ \\
$D^{2} \Sigma^{+}$ & $0.10(7)$ & $0.73(7)$ & $0.22(7)$ & $0.18(8)$ & $\ldots$ & $\ldots$ & $\ldots$ & $\ldots$ & $\ldots$ & $\ldots$ \\
$E^{2} \Pi$ & $0.52(7)$ & $0.42(7)$ & $0.10(8)$ & $0.86(7)$ & $\ldots$ & $\ldots$ & $\ldots$ & $\ldots$ & $\ldots$ & $\ldots$ \\
$1^{2} \Delta$ & $\ldots$ & $\ldots$ & $0.34(8)$ & $\ldots$ & $\ldots$ & $\ldots$ & $\ldots$ & $\ldots$ & $\ldots$ & $\ldots$ \\
$5^{2} \Sigma^{+}$ & $0.20(7)$ & $0.14(7)$ & $0.24(8)$ & $0.43(7)$ & $0.66(5)$ & $0.62(5)$ & $\ldots$ & $\ldots$ & $\ldots$ & $\ldots$ \\
$6^{2} \Sigma^{+}$ & $0.41(8)$ & $0.26(7)$ & $0.10(7)$ & $0.43(6)$ & $0.50(7)$ & $0.16(7)$ & $\ldots$ & $\ldots$ & $\ldots$ & $\ldots$ \\
$3^{2} \Pi$ & $0.18(8)$ & $0.33(6)$ & $0.13(7)$ & $0.21(7)$ & $0.13(7)$ & $0.28(6)$ & $0.44(6)$ & $0.31(6)$ & $\ldots$ & $\ldots$ \\
$4^{2} \Pi$ & $0.72(7)$ & $0.35(7)$ & $0.24(6)$ & $0.38(6)$ & $0.28(6)$ & $0.22(7)$ & $0.15(5)$ & $0.68(5)$ & $0.22(7)$ & $1.00(7)$ \\
$7^{2} \Sigma^{+}$ & $0.12(6)$ & $0.62(5)$ & $0.20(7)$ & $0.16(7)$ & $0.28(6)$ & $0.40(6)$ & $\ldots$ & $\ldots$ & $\ldots$ & $\ldots$ \\
$5^{2} \Pi$ & $0.54(6)$ & $0.59(6)$ & $0.32(7)$ & $0.11(5)$ & $0.35(7)$ & $0.73(7)$ & $0.11(7)$ & $0.38(7)$ & $0.20(6)$ & $0.13(7)$ \\
$2^{2} \Delta$ & $\cdots$ & $\ldots$ & $0.13(7)$ & $\ldots$ & $\ldots$ & $0.13(7)$ & $0.68(7)$ & $\ldots$ & $\ldots$ & $0.52(7)$ \\
$3^{2} \Delta$ & $\ldots$ & $\ldots$ & $0.14(7)$ & $\ldots$ & $\ldots$ & $0.50(7)$ & $0.62(7)$ & $\ldots$ & $\ldots$ & $0.64(7)$ \\
$1^{2} \Phi$ & $\ldots$ & $\ldots$ & $\ldots$ & $\ldots$ & $\ldots$ & $\ldots$ & $0.17(8)$ & $\ldots$ & $\ldots$ & $\ldots$ \\
$8^{2} \Sigma^{+}$ & $0.13(8)$ & $0.23(7)$ & $0.12(5)$ & $0.21(6)$ & $0.74(6)$ & $0.36(5)$ & $\ldots$ & $\ldots$ & $\ldots$ & $\ldots$ \\
$9^{2} \Sigma^{+}$ & $0.27(7)$ & $0.65(6)$ & $0.42(5)$ & $0.26(7)$ & $0.53(7)$ & $0.80(7)$ & $\ldots$ & $0.39(7)$ & $0.18(6)$ & $0.14(6)$ \\
$10^{2} \Sigma^{+}$ & $0.59(7)$ & $0.47(6)$ & $0.34(5)$ & $0.85(6)$ & $0.39(6)$ & $0.32(5)$ & $\ldots$ & $0.18(5)$ & $0.56(6)$ & $0.32(6)$ \\
$6^{2} \Pi$ & $0.26(7)$ & $0.34(4)$ & $0.85(6)$ & $0.59(6)$ & $0.13(7)$ & $0.20(7)$ & $0.12(6)$ & $0.39(5)$ & $0.48(6)$ & $0.77(6)$ \\
$11^{2} \Sigma^{+}$ & $0.13(4)$ & $0.16(4)$ & $0.76(7)$ & $0.12(6)$ & $0.14(5)$ & $0.12(6)$ & $\ldots$ & $0.17(4)$ & $0.82(4)$ & $0.14(7)$ \\
$7^{2} \Pi$ & $0.63(6)$ & $0.38(6)$ & $0.12(7)$ & $0.59(6)$ & $0.55(6)$ & $0.26(7)$ & $0.90(5)$ & $0.17(7)$ & $0.65(5)$ & $0.25(6)$ \\
$12^{2} \Sigma^{+}$ & $0.42(8)$ & $0.19(7)$ & $0.28(7)$ & $0.88(6)$ & $0.13(6)$ & $0.28(7)$ & $\ldots$ & $0.24(6)$ & $0.38(6)$ & $0.38(7)$ \\
$8^{2} \Pi$ & $0.19(8)$ & $0.67(7)$ & $0.13(6)$ & $0.11(7)$ & $0.33(6)$ & $0.38(5)$ & $0.13(7)$ & $0.35(6)$ & $0.17(6)$ & $0.17(6)$ \\
\hline \hline
\end{tabular}

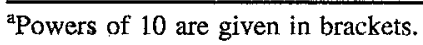

and ${ }^{2} \Phi$ states (cf. Table I). The character of all the excited states calculated at 2.5 bohr is Rydberg. However for most of these states it is not possible to attach atomic labels, on the basis of the character of the MO because they show mixtures of atomic characters, as allowed by the molecular symmetry. This is particularly truc for thc ${ }^{2} \Pi$ states, where all the states are obtained as mixtures of $p, d$, and $f$ diffuse orbitals. The labels listed next to the states in Table I refer to the corresponding dissociation limits, $\mathrm{H}^{*}+\mathrm{Ar}$ and $\mathrm{Ar}^{*}\left({ }^{1,3} \mathrm{P}\right)+\mathrm{H}$.

In addition to the energies, the radiative lifetimes of the Rydberg states are also listed in Table I. They have been obtained by considering all the transitions to lower states up to $3^{2} \Pi$, for which the dipole transition probabilities are given in Table II. As the data in Table II show, for most states there are several transitions which must be summed in order to obtain a good estimate of their radiative lifetimes. Thus, for example, our previous value for the radiative lifetime of $D^{2} \Sigma^{+}$, based on transitions to $X^{2} \Sigma^{+}, A^{2} \Sigma^{+}$, and $B{ }^{2} \Pi$ states, was $496 \mathrm{~ns}$ while the present value which includes also the transition to $C^{2} \Sigma^{+}$(i.e., all possible radiative transitions) has the value 36.0 ns. The radiative lifetimes listed for the $7^{2} \Sigma^{+}$state or higher ${ }^{2} \Sigma^{+}$states and $5^{2} \Pi$ or higher ${ }^{2} \Pi$ states do not include all possible transitions to lower states since lower states only up to $3^{2} \Pi$ have been included. For the lower-lying states, the present results should be converged to within about $20 \%-30 \%$ which is the expected variation which may be caused by changes in the $\mathrm{AO}$ basis set and in the CI procedure.

Of the radiative lifetimes listed in Table I, experimental values exist for the $B{ }^{2} \Pi$ state, where it has been reported as $14.3 \pm 5 \mathrm{~ns}$ and also as $-18 \mathrm{~ns}$, and for the $E^{2} \Pi$ state also mentioned in the Introduction, where rather interesting re- sults have been reported. The lifetime of the $Q$ branch (of the $E^{2} \Pi \rightarrow A^{2} \Sigma^{+}$transition) in ArH is reported as $39.8 \mathrm{~ns} \pm 4.9$ (for $N \leqslant 16$ ), while in $\operatorname{ArD}$, for the $P$ and $R$ branches $(N \leqslant 19)$ it is listed as $42.0 \pm 3.6 \mathrm{~ns}$, where in both the above cases no dependence of the lifetime on the rotational level $N$ is found. On the basis of the above data it could be concluded that there is good agreement between the theoretical and experimental radiative lifetimes of $E^{2} \Pi$. However, the reported lifetime of the $Q$ branch of the $E^{2} \Pi \rightarrow A^{2} \Sigma^{+}$transition in $\operatorname{ArD}$ is $60.6 \pm 1.1 \mathrm{~ns}$ for $N \leqslant 19$ and $49.8 \pm 4.7 \mathrm{~ns}$ for $N \approx 30$. Thus the discrepancy between the theoretical value and the experimental lifetime of the $Q$ branch in $\operatorname{ArD}$ is rather large especially for $N \leqslant 19$. If the rationalization of Wunderlich et $a l^{8}{ }^{8}$ is accepted, i.e., that the shorter lifetimes of the $P$ and $R$ brances are a result of predissociation then the lifetime of the $Q$ branch would be closer to the radiative lifetime of the state. For this reason some additional calculations devoted to the radiative lifetime of $E^{2} \Pi$ have been performed in order to estimate the convergence of the theoretical value. Different calculations yield slightly different lifetimes for $E^{2} \Pi$. The previous value of $37.5 \mathrm{~ns}$ for the radiative lifetime of ArH had been obtained for transitions to $X^{2} \Sigma^{+}, A^{2} \Sigma^{+}$, and $B{ }^{2} \Pi$ only. ${ }^{9}$ The value of $35.3 \mathrm{~ns}$ of the present work (cf. Table I) includes also the transition to $C^{2} \Sigma^{+}$. A value of $32 \mathrm{~ns}$ is obtained by a calculation with a lower threshold $(2.5 \mu \mathrm{s})$ for the ${ }^{2} I$ states while a calculation with a split-Rydberg basis set (i.e., with double the number of Rydberg functions) resulted in a value of $34 \mathrm{~ns}$. All the above values are very close to each other. In all these calculations, the CI procedure involved selection for many roots (cf. previous section and Ref. 9). A value of 29 ns has been obtained in the calculations of the present work, involving 


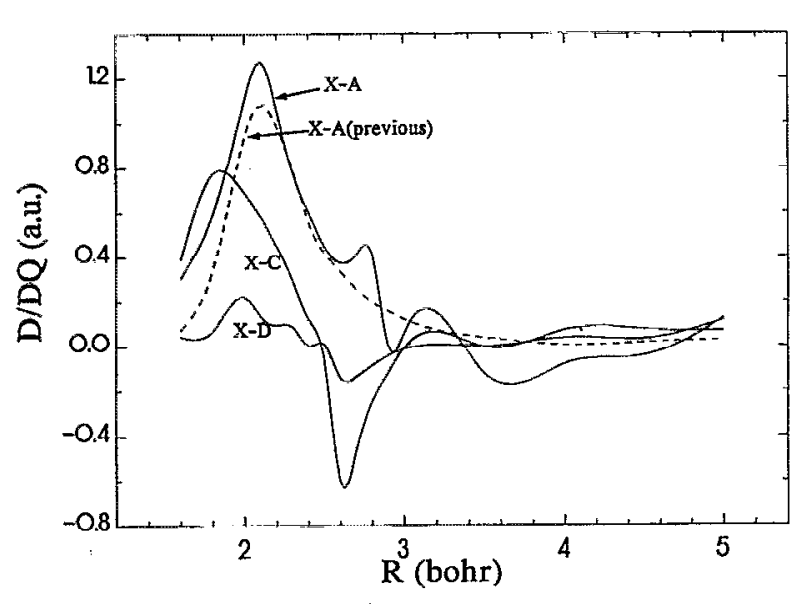

FIG. 2. Radial coupling matrix elements over the electronic wave functions of the $A^{2} \Sigma^{+}, C^{2} \Sigma^{+}$, and $D^{2} \Sigma^{+}$states of ArH with $X^{2} \Sigma^{+}$. Solid lines, present work; dashed, previous work.

four ${ }^{2} \Sigma$, one ${ }^{2} \Delta$, and three ${ }^{2} \Pi$ roots, described above, in the first part of Sec. II. It thus appears that the selection procedure and in particular the number of roots involved affects the calculated radiative lifetime significantly. In any case, the above results yield a radiative lifetime for $E^{2} \Pi$ between 32 and $38 \mathrm{~ns}$, as obtained from the vertical transition at $R^{\prime}$ of 2.5 bohr, and do not support the reported experimental lifetime of the $Q$ branch, of $60.6 \pm 1.1 \mathrm{~ns}$.

\section{B. Radial coupling matrix elements}

The calculated radial coupling matrix elements for the $X-A, X-C$, and $X-D$ interactions have been plotted in Fig. 2 , where for $X-A$ the values calculated previously have been also plotted for comparison. As shown in Fig. 2, the $X^{2} \Sigma^{+}-A^{2} \Sigma^{+}$radial coupling matrix elements of the present work are not identical to the previous. The main differences is a $20 \%$ increase of the peak and an oscillatory behavior of the present results at $R>2.6 \mathrm{bohr}$, which were not present in the 3-root calculation. ${ }^{9}$ A lowering of the threshold of the present calculations to $1 \mu \mathrm{H}$ for a few testcalculations did not alter the above picture significantly. From this it may be concluded that the differences between the previous and the present radial coupling matrix elements, are mainly due to the presence of more roots in the present work, which allow configurations in the $\mathrm{CI}$ space which might have been omitted on the basis of an energy criterion for the three roots only. The $X^{2} \Sigma^{+}-C^{2} \Sigma^{+}$interaction (cf. Fig. 2) is smaller than the $X-A$ at small $R$ but has larger magnitude than the $X-A$ at $R$ around 2.6, while the $X-D$ interaction has generally small magnitude. The above matrix elements lead to the predissociation of the $A, C$, and $D$ states by $X$ and the calculated widths of the states are listed in Table III. These widths have been calculated by a FermiGolden rule method ${ }^{14,16}$ where only the $(\partial / \partial R)$ radial coupling has been included. Previous work ${ }^{9}$ has shown that the $\left(\partial^{2} / \partial R^{2}\right)$ term has a negligible influence and since the $\left(\partial^{2} / \partial R^{2}\right)$ interaction is even more sensitive to the computational procedure than the first derivative, it does not make good sense to include it. The calculated widths listed in Table
TABLE III. Calculated widths $\left(\mathrm{cm}^{-1}\right)$ for the rotational level $N=0$ of different vibrational levels $v$ in the $A^{2} \Sigma^{+}, C^{2} \Sigma^{+}$, and $D^{2} \Sigma^{+}$electronic states of ArH and ArD.

\begin{tabular}{llllllll}
\hline \hline & \multicolumn{3}{c}{$\operatorname{ArH}$} & \multicolumn{3}{c}{$\operatorname{ArD}$} \\
\cline { 2 - 4 } \cline { 6 - 8 }$n$ & \multicolumn{1}{c}{$A^{2} \Sigma^{+}$} & $C^{2} \Sigma^{+}$ & $D^{2} \Sigma^{+}$ & & $A^{2} \Sigma^{+}$ & $C^{2} \Sigma^{+}$ & $D^{2} \Sigma^{+}$ \\
\hline 0 & $0.367(0.15)^{\mathrm{a}}$ & 0.345 & 0.002 & $0.014(0.02)$ & 0.009 & 0.004 \\
1 & $0.930(0.43)$ & 1.182 & 0.024 & $0.079(0.04)$ & 0.032 & 0.009 \\
2 & 1.310 & 0.394 & 0.033 & 0.213 & 0.098 & 0.011 \\
3 & 2.240 & 1.247 & 0.032 & 0.379 & 0.205 & 0.012 \\
4 & 2.352 & 0.884 & 0.045 & 0.526 & 0.288 & 0.012 \\
5 & 2.677 & 0.832 & 0.066 & 0.683 & 0.337 & 0.011 \\
\hline \hline
\end{tabular}

${ }^{a}$ Values in brackets from previous calculations (Ref. 9).

III show that the $A$ and $C$ states have large widths while the $D$ state does not. Between $\operatorname{ArH}$ and $\operatorname{ArD}$, there is a dramatic reduction in the widths of the $A$ and $C$ states while there is no significant change in the widths of the $D$ state. It might also be noted that the previously mentioned differences in the calculated $X^{2} \Sigma^{+}-A^{2} \Sigma^{+}$radial coupling matrix elements of the previous ${ }^{9}$ and the present work, results in widths for the $A^{2} \Sigma^{+}$state of ArH larger by about a factor of 2 calculated in the present work than previously (cf. values in brackets in Table III).

A measure of the accuracy of the numbers listed in the above table is given by comparison with the available experimental data. The most recently ${ }^{7}$ quoted experimental widths for the $v=0$ level of the $A$ state are $1 \mathrm{~cm}^{-1}$ for $\mathrm{ArH}$ (previous ${ }^{6}$ value $2 \mathrm{~cm}^{-1}$ ) and $0.03 \mathrm{~cm}^{-1}$ for $\mathrm{ArD}$ (previous ${ }^{6}$ value $<0.05 \mathrm{~cm}^{-1}$ ). In terms of the predissociation lifetimes, theory and experiment obtain a lifetime of 14 and $5 \mathrm{ps}$, respectively, for the $v=0$ level of $A^{2} \Sigma^{+}$in ArH and 0.5 and $0.1 \mathrm{~ns}$, respectively, in ArD. Such agreement between theory and experiment is acceptable considering the difficulty of the task. ${ }^{3,9}$ The predissociation lifetimes of the $v=0$ level of the $C^{2} \Sigma^{+}$state are $15 \mathrm{ps}$ in $\mathrm{ArH}$ and $0.6 \mathrm{~ns}$ in $\mathrm{ArD}$, i.e., they are similar to the values found for $A^{2} \Sigma^{+}$. However, as the $X^{2} \Sigma^{+}-D^{2} \Sigma^{+}$coupling matrix elements (cf. Fig. 2) and the resulting widths (cf. Table III) show, the predissociation rates in the $D^{2} \Sigma^{+}$state are smaller than in the $A$ and $C$ states. Accordingly, the predissociation lifetime of the $v=0$ level is obtained as $2.8 \mathrm{~ns}$ in $\mathrm{ArH}$ and $1.4 \mathrm{~ns}$ in ArD. Thus within the expected precision of the present results, similar predissociation rates are predicted for $D^{2} \Sigma^{+}$in ArII and in ArD. This is the first such example encountered in the rare-gas hydrides, where at least for the $A$ state, significantly lower predissociation rates are found in the deuterated compounds. $9,14,16$

A comparison of the predissociation lifetimes with the radiative lifetimes (cf. Table I) shows that for the $A^{2} \Sigma^{+}$ state in both ArH and ArD predissociation is by far the predominant mode of decay and similarly for the $C^{2} \Sigma^{+}$state in ArH. However for the $C^{2} \Sigma^{+}$state in $\operatorname{ArD}$ and the $D^{2} \Sigma^{+}$in both ArH and ArD predissociation is faster than radiative decay by a factor of about 10 and thus observation of radiative transitions from these states is possible. Indeed, as noted earlier, radiative transitions $p\left(C^{2} \Sigma^{+}, B^{2} \Pi\right) \rightarrow A^{2} \Sigma^{+}$in ArD have been reported by Dabrowski et al. ${ }^{7}$

In Fig. 3, there are plots of $(\partial / \partial R)$ matrix elements between the bound ${ }^{2} \Sigma^{+}$states. As shown they have large mag- 


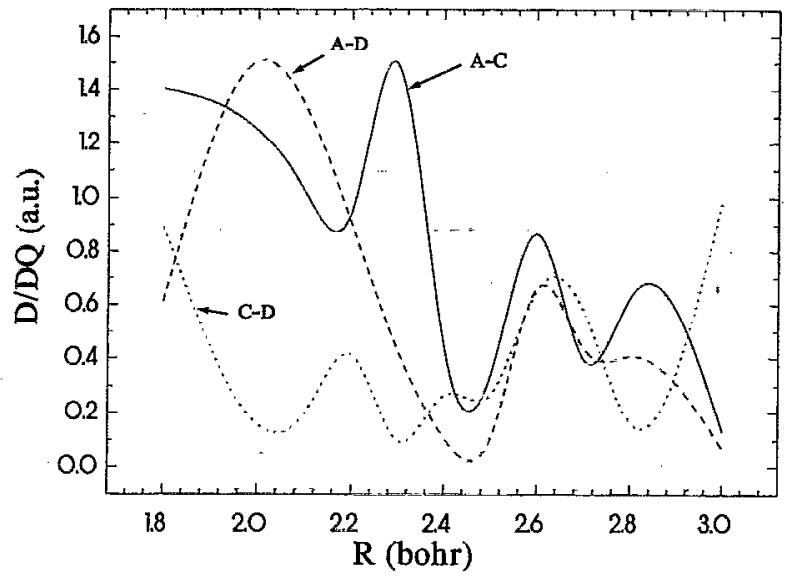

FIG. 3. Radial coupling matrix elements over pairs of bound ${ }^{2} \Sigma^{+}$states of ArH.

nitudes, comparable to those occurring with the $X^{2} \Sigma^{+}$state (cf. Fig. 2). The implication of this is the possibility of rotational perturbations of the spectra whenever there is nearcoincidence of the vibrational levels ${ }^{17}$ and possibly predissociation when the coincidence involves the vibrational continuum of the lower-lying states. Such processes have been present in $\mathrm{HeH}$, where the $C^{2} \Sigma^{+}-D^{2} \Sigma^{+}$interaction gave rise to rotational perturbations in the $D^{2} \Sigma^{+} \rightarrow A^{2} \Sigma^{+}$ spectrum ${ }^{15,18}$ whilc levels with $N>14$, of the $C^{2} \Sigma^{+}$state are predissociated by $A^{2} \Sigma^{+} .16$ In the system at hand, the Rydberg states lie closer together and predissociation of the higher states, $C^{2} \Sigma^{+}$and $D^{2} \Sigma^{+}$is not possible for the lower vibrational levels even at high rotational levels. In particular, for levels $v=0$ and 1 of $D^{2} \Sigma^{+}$even for $N=30$ the energy lies below the continuum of the $A^{2} \Sigma^{+}$state. On the other hand, the proximity of the curves may lead to rotational perturbations of the Rydberg spectra involving these states. A very important factor for the magnitude of the shifts of the energy levels is the degree of coincidence of the unperturbed levels. ${ }^{17}$ On the basis of the theoretical energies, coincidences may exist between the rotational levels $v \geqslant 3$ of $A^{2} \Sigma^{+}$in $\operatorname{ArH}(v \geqslant 4$ in $\mathrm{ArD})$ and rotational levels of $v>0$ of $C^{2} \Sigma^{+}$. Similarily, rotational levels of $V \geqslant 6, A^{2} \Sigma^{+}$in $\mathrm{ArH}(v \geqslant 8$ in $\mathrm{ArD})$ may overlap with rotational levels of $v \geqslant 0, D^{2} \Sigma^{+}$, while rotational levels of $v \geqslant 4, C^{2} \Sigma^{+}$in ArH $(v \geqslant 5$ in ArD) may coincide with rotational levels of $v \geq 0, D^{2} \Sigma^{+}$.

It is possible to obtain the theoretical coupling between the vibrational levels, for pairwise interactions using the offdiagonal matrix element

$$
H_{i j}=-\frac{1}{2 \mu}\left\langle\chi_{v x}^{i}\left|2 A(R) \frac{\partial}{\partial R}\right| \chi_{v y}^{j}\right\rangle
$$

where $x$ and $y$ refer to the different electronic states $A, C$, or $D$, while $\chi$ are vibrational wave functions. $A(R)$ is an analytic fit to the coupling matrix elements of $(\partial / \partial R)$ over the electronic wave functions (cf. Fig. 3). In a $2 \times 2$ calculation (i.e., including only the two coincident vibrational-rotational
TABLE IV. Interaction matrix elements ${ }^{\mathrm{a}}$ over levels near-coincident with the $v=0$ and $v=1(N=0)$ levels of $C^{2} \Sigma^{+}$and $D^{2} \Sigma^{+}$in ArH and ArD.

\begin{tabular}{|c|c|c|c|}
\hline & & $\cdots \quad$ ArH & ArD \\
\hline \multirow[t]{2}{*}{$C^{2} \Sigma^{+}$} & $v=0$ & $\begin{array}{l}-0.8806(-3) \\
v=3, A^{2} \Sigma^{+b}\end{array}$ & $\begin{array}{l}-0.2481(-3) \\
v=4, A^{2} \Sigma^{+}\end{array}$ \\
\hline & $v=1$ & $\begin{array}{c}0.8902(-3) \\
v=4, A^{2} \Sigma^{+}\end{array}$ & $\begin{array}{c}0.2719(-3) \\
v=5, A^{2} \Sigma^{+}\end{array}$ \\
\hline \multirow[t]{4}{*}{$D^{2} \Sigma^{+}$} & $v=0$ & $\begin{array}{l}-0.3377(-3) \\
v=6, A^{2} \Sigma^{+}\end{array}$ & $\begin{array}{c}0.7700(-4) \\
v=8, A^{2} \Sigma^{+}\end{array}$ \\
\hline & $v=1$ & $\begin{array}{c}0.2718(-3) \\
0=7, A^{2} \Sigma^{+}\end{array}$ & $\begin{array}{c}0.1457(-3) \\
v=9, A^{2} \Sigma^{+}\end{array}$ \\
\hline & $v=0$ & $\begin{array}{c}0.3769(-4) \\
v=4, C^{2} \Sigma^{+}\end{array}$ & $\begin{array}{c}0.2264(-5) \\
0=5, C^{2} \Sigma^{+}\end{array}$ \\
\hline & $v=1$ & $\begin{array}{c}0.1543(-3) \\
v=5, C^{2} \Sigma^{+}\end{array}$ & $\begin{array}{c}0.5566(-4) \\
v=6, C^{2} \Sigma^{+}\end{array}$ \\
\hline
\end{tabular}

"In hartree, values in brackets are powers of ten.

"The "partner" level involved in the interaction matrix element.

levels), for a situation of exact coincidence, the magnitude of the coupling (1), gives the resulting shift of the energy level. $^{17}$

In the present calculations, the $2 \times 2$ model is generalized by allowing all bound vibrational levels with $N=0$ of each state to interact. A secular equation is set up whereby the diagonal matrix elements are the unperturbed vibrational energies and the off-diagonal matrix elements are as in Eq. (1). Diagonalization gives the resulting perturbed energy levels. However, the magnitude of the perturbation will be very sensitive to the actual energy spacing and for experimentally observable shifts, the experimental relative energies must be employed.

A gencral multistate treatment would require setting up such a secular equation over all the electronic states with all the possible off-diagonal interactions in order to take them into account simultaneously. Such an approach is not followed here but a secular equation is set up for each pair of electronic states, where as noted above, all the bound vibrational levels are allowed to interact via the radial coupling. In Table IV, the interaction matrix elements for the $v=0$ and $v=1$ (with $N=0$ ) levels of $C^{2} \Sigma^{+}$and $D^{2} \Sigma^{+}$with coincident levels of $A^{2} \Sigma^{2}$ (for both $C$ and $D$ ) and $C^{2} \Sigma^{+}$(for $D^{2} \Sigma^{+}$) are given. As shown in Table IV, the interaction matrix elements have larger magnitudes for the $A-C$ and $A-D$ pairs than the $C-D$. Furthermore, generally smaller interactions are predicted for $\operatorname{ArD}$ than for ArH. As noted above, the listed matrix elements would give the shift of the levels (in a two-level model) in a situation of exact coincidence. However, the present calculations obtain significant interaction matrix elements over noncoincident pairs of levels as well and the solution of the secular equations produces shifts slightly different from what would be expected on the basis of a two-state model alone. Thus the predicted shifts for the $v=0, N=0$ and $v=1, N=0$ levels of $C^{2} \Sigma^{+}$due to the $A-C$ interaction are 84 and $202 \mathrm{~cm}^{-1}$, respectively in ArH and -18 and $-3 \mathrm{~cm}^{-1}$, respectively in ArD. The $A-D$ interaction results in shifts of 70 and $-32 \mathrm{~cm}^{-1}$ for the $v=0$, $N=0$ and $v=1, N=0$ levels of $D^{2} \Sigma^{+}$, respectively in ArH and the corresponding shifts of -2 and $-11 \mathrm{~cm}^{-1}$ in ArD. 


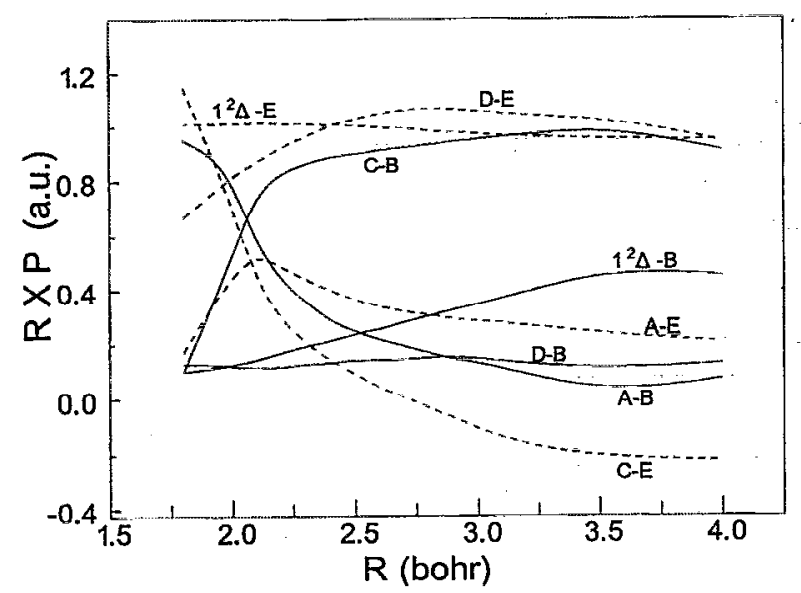

FIG. 4. Rotational-electronic coupling matrix elements over the electronic wave functions of bound ${ }^{2} \Sigma-{ }^{2} \Pi$ and ${ }^{2} \Pi-{ }^{2} \Delta$ Rydberg states of $A r H$.

The $C-D$ interaction gives rise to shifts of -5 and $10 \mathrm{~cm}^{-1}$ for the $n=0, N=0$ and $v=1, N=0$ levels of $D^{2} \Sigma^{+}$in ArH and shifts of -2 and $-4 \mathrm{~cm}^{-1}$ in ArD. The above have been obtained using the theoretical energy levels. If the potential of the $C^{2} \Sigma^{+}$is shifted upwards by $1070 \mathrm{~cm}^{-1}$ as would be required to match the experimental $p\left({ }^{2} \Sigma^{+},{ }^{2} \Pi\right) \rightarrow A^{2} \Sigma^{+}$energy difference ${ }^{7}$ the shifts are altered dramatically. For the $v=0, N=0$ of $C^{2} \Sigma^{+}$in ArH the predicted shift is -62 $\mathrm{cm}^{-1}$ and for the $v=1, N=0$ level it is $-43 \mathrm{~cm}^{-1}$. In ArD, the corresponding shifts are now -21 and $-9 \mathrm{~cm}^{-1}$, respectively.

From the above analysis it may be concluded that the bound-bound radial coupling interaction between the ${ }^{2} \mathbf{\Sigma}^{+}$ states of ArH would lead to observable rotational perturbations in the $C \rightarrow A$ and $D \rightarrow A$ Rydberg spectra of ArH, if such were observed. In $\operatorname{ArD}$, only for the $C^{2} \Sigma^{+}$state there might be an appreciable effect. Perhaps such perturbations of the rotational levels of $C^{2} \Sigma^{+}$give rise to some of the complications encountered in the spectrum of ArD which involves this state in conjunction with $B{ }^{2} \Pi$ referred to as a $p\left({ }^{2} \Sigma^{+},{ }^{2} \Pi\right)$ state.

\section{Rotational-electronic coupling}

The rotational-electronic coupling matrix elements connecting the ground state, $X^{2} \Sigma^{+}$, with the ${ }^{2} \Pi$ Rydberg states have been presented elsewhere and it was shown that such interactions are small and do not lead to predissociation of the ${ }^{2} \Pi$ states. In the present work only the bound ${ }^{2} \Sigma^{+}-{ }^{2} \Pi$ interactions shall be considered, plots of which are presented in Fig. 4. As shown in Fig. 4, the rotational-electronic coupling matrix elements are generally small except for those of the pairs $C^{2} \Sigma^{+}-B^{2} \Pi, D^{2} \Sigma^{+}-E^{2} \Pi$, and $E^{2} \Pi-1^{2} \Delta$ which have values near 1.0. These results are in accord with the experimental analyses. As noted earlier, the $C^{2} \Sigma^{+}$and $B{ }^{2} \Pi$ states are considered as forming a $p(\Sigma, \Pi)$ complex while the $D^{2} \Sigma^{+}, E^{2} \Pi$, and $1^{2} \Delta$ state might be thought of as a " $d$ " complex. ${ }^{10}$

The interaction of the above pairs of states over different vibrational-rotational levels is obtained in a manner similar to that employed for the radial coupling interactios (Sec. III B), where in this case the operator for the off-diagonal matrix elements is given by

$$
-\frac{1}{2 \mu} \sqrt{(N \pm \Lambda)(N+1 \mp \Lambda)} \frac{\left\langle\Psi_{\Lambda}(R)|L \pm| \Psi_{\Lambda \mp 1}(R)\right\rangle}{R^{2}},
$$

where $N$ is the rotational number, $\Lambda$ has the usual values 0,1 , 2 for $\Sigma, \Pi$, and $\Delta$ states respectively, and the upper signs correspond to the $L_{+}$and the lower to the $L_{-}$operator. As Eq. (2) shows, the interaction is directly dependent on $N$ and thus it is necessary to carry out the calculation for different rotational levels. In the present work, the interaction of three vibrational levels $(v=0,1,2)$ for 30 rotational levels has been considered, for each pair of electronic states. The results of these treatments are as follows:

\section{1. $C^{2} \Sigma^{+}-B^{2} \Pi$ interaction}

For rotational level $N=1$, in $v=0$ the $C-B$ interaction in ArH has a magnitude of $18 \mathrm{~cm}^{-1}$, and increases with $N$ as expected. Thus for $N=20$ the interaction has the magnitude $250 \mathrm{~cm}^{-1}$. In $\mathrm{ArD}$, the computed magnitudes for the $C-B$ interaction are smaller than in ArH by a factor near 2, which is the ratio of the reduced mass of ArD over that of ArH. This type of interaction produces strong perturbations in the rotational levels and is consistent with the formation of a complex $(\Sigma, \Pi)$ state such as the $p(\Sigma, \Pi)$ complex invoked by Dabrowski et al. ${ }^{7}$ The mixing of the two states is indicated in their coefficients following the solution of the secular equation. Thus in ArH the coefficients are 0.9922 and 0.1248 corresponding to the $N=1, v=0$ unperturbed levels of the $B$ and $C$ states, respectively, when the theoretical energies are employed. However, if the potential energy curve of the $C^{2} \Sigma^{+}$state is shifted downwards by 0.00068 hartree so as to bring the $v=0, N=1$ levels of $C^{2} \Sigma^{+}$and $B^{2} \Pi$ in coincidence, the corresponding mixing coefficients are 0.7920 and 0.6105 , respectively, and the mixing increases with $N$. For $N=8$ there is a 50:50 mixture of the two states, in accord with a complex $(\Sigma, \Pi)$ state. It should be noted, however, that the above interaction would affect only the $\Pi^{+}$component of the $B^{2} \Pi$ state and leave $\Pi^{-}$unaffected, whereas in a $p(\Sigma, \Pi)$ complex the $\Sigma$ and $\Pi$ (thus $\Pi^{+}$and $\mathrm{II}^{-}$) characteristics would not be evident. ${ }^{10}$

\section{2. $D^{2} \Sigma^{+}-E^{2} \Pi$ and $E^{2} \Pi-1^{2} \Delta$ interactions in ArH and ArD}

The magnitudes of the rotational electronic-coupling for different rotational levels of the above pairs of states are similar to these obtained for the $C-B$ interaction (see above). Again, the closeness of the potentials of the $D$ and $E$ states (cf. Table I) allows for the possibility of coincidences of the levels of the two states and a strong mixing of the two states. With regard to the $1^{2} \Delta$ state, the calculated energy is above that of the $E^{2} \Pi$ state (cf. Table I) and it would seem that a coincidence of the rotational levels of ${ }^{1} \Delta$ with $E^{2} \Pi$ is less probable, although it cannot be excluded. The accuracy of the electronic calculations is not such that would allow a definite conclusion regarding the relative positions of these states. In any case, the results of the present calculations 
indicate that there are strong interactions between the $D^{2} \Sigma^{+}$ and $E^{2} \Pi$ as well as the $E^{2} \Pi$ and the $1^{2} \Delta$ states, sufficient for the formation of a complex $(\Sigma, \Pi, \Delta)$ state (for $N \geqslant 2$ ), depending on the degree of coincidence of the rotational levels. Again, only the $\Pi^{+}$component would mix with the $D^{2} \Sigma^{+}$state while the interaction with $1^{2} \Delta$ would affect both the $\Pi^{+}$and the $\Pi^{-}$components, assuming that $\Lambda$ is a good quantum number for these states. The existence of a strong $D^{2} \Sigma^{+}-E^{2} \Pi$ interaction would be in accord with the assumed depletion of population of $E^{2} \Pi^{+}$by predissociation via $D^{2} \Sigma^{+}$. However, this would still leave us with a large discrepancy between the theoretical and the experimental value of the lifetime of $E^{2} \Pi$.

\section{CONCLUSIONS}

In the present work radiative lifetimes of Rydberg states of $\mathrm{ArH}$ and $\mathrm{ArD}$ up to $4 f$ have been presented and it was found that for most states there exist more than one important radiative channel of decay. Predissociation of the ${ }^{2} \Sigma^{+}$ states by $X^{2} \Sigma^{+}$has been found to be the predominant mode of decay for $A^{2} \Sigma^{+}$in ArH and in ArD and for the $C^{2} \Sigma^{+}$ state in ArH, while for the $C^{2} \Sigma^{+}$state in ArD and the $D^{2} \Sigma^{+}$in both $A r H$ and $A r D$ radiative decay is slower than predissociation by about a factor of 10 . In addition to the interactions with the ground state, bound-bound interactions between the Rydberg states have been also found to be significant. In particular, radial coupling interactions of $C^{2} \Sigma^{+}$ and $D^{2} \Sigma^{+}$with $A^{2} \Sigma^{+}$are strong but the actual perturbations on the spectra will depend on the actual relative energy of the states, which cannot be obtained with the required accuracy for such perturbations, by the ab initio calculations. Strong rotational-electronic interactions have been found for the states $C^{2} \Sigma^{+}$with $B^{2} \Pi$ and $E^{2} \Pi$ with $D^{2} \Sigma^{+}$and $1^{2} \Delta$ indicating large perturbations and mixing of the above states. These results are consistent with the formation of a $(\Sigma, \Pi)$ complex between the $B^{2} \Pi$ and $C^{2} \Sigma^{+}$, such as invoked by Dabrowski et al. ${ }^{7}$ while an analogous type of $(\Sigma, \Pi, \Delta)$ complex might be visualized for the $D^{2} \Sigma^{+}, E^{2} \Pi$, and $1^{2} \Delta$ states.

${ }^{1}$ G. Herzberg, Annu. Rev. Phys. Chem. 38, 27 (1987).

${ }^{2}$ G. Theodorakopoulos, S. C. Farantos, R. J. Buenker, and S. D. Peyerimhoff, J. Phys. B 17, 1453 (1984).

${ }^{3}$ M. C. Van Hemert, H. Dohman, and S. D. Peyerimhoff, Chem. Phys. 110, 55 (1986).

${ }^{4}$ G. Theodorakopoulos, I. D. Petsalakis, and R. J. Buenker, Mol. Phys. 71, 1055 (1990).

${ }^{5}$ R. H. Lipson, Mol. Phys. 65, 1217 (1988).

${ }^{6} \mathrm{~J}$. W. C. Johns, J. Mol. Spectrosc. 36, 488 (1970).

${ }^{7}$ I. Dabrowski, G. Dilonardo, G. Herzberg, J. W. C. Johns, D. A. Sadovskii, and M. Vervloct, J. Chem. Phys. 97, 7093 (1992).

${ }^{8}$ C. Wunderlich, V. Betz, R. Bruckmeier, and H. Figger, J. Chem. Phys. 98, 9362 (1993).

${ }^{9}$ I. D. Petsalakis and G. Theodorakopoulos, J. Phys. B 25, 5353 (1992).

${ }^{10}$ I. Dabrowski (private communication).

${ }^{\mathrm{L}}$ R. J. Buenker, S. D. Peyerimhoff, and W. Butscher, Mol. Phys. 35, 771 (1978).

${ }^{12}$ R. J. Buenker, Studies in Physical and Theoretical Chemistry (Current Aspects of Quantum Chemistry), edited by R. Carbo (Elsevier, Amsterdam, 1981), Vol. 21, p. 17; R. J. Buenker and R. Phillips, J. Mol. Struct. Theochem 123, 291 (1985).

${ }^{13}$ G. Hirsch, P. J. Bruna, R. J. Buenker, and S. D. Peyerimhoff, Chem. Phys. 45, 335 (1980).

${ }^{14}$ I. D. Petsalakis, G. Theodorakopoulos, and R. J. Buenker, Phys. Rev. A 38, 4004 (1988).

${ }^{15} \mathrm{G}$. Theodorakopoulos, I. D. Petsalakis, and R. J. Buenker, Chem. Phys. Lett. 148, 285 (1988).

${ }^{16}$ I. D. Petsalakis, G. Theodorakoopoulos, and R. J. Buenker, J. Chem. Phys. 92, 4920 (1990).

${ }^{17} \mathrm{G}$. Herzberg, Spectra of Diatomic Molecules (Van Nostrand, Princeton, 1950).

${ }^{18}$ R. L. Brooks and B. G. Nickel, Chem. Phys. Lett. 139, 503 (1987). 